\title{
CONFLICTING EVIDENCE ON THE COMPOSITION OF AM STARS
}

\author{
CLAUDE VAN'T VEER \\ Institut d'Astrophysique de Paris, France \\ and \\ CLAUDE BURKHART \\ Observatoire de Lyon, France
}

Discordance à propos de la composition d'étoiles Am

Résumé.

Les étoiles Am sont des objets typiques qui posent notamment des problèmes au niveau de la relation entre la classification et les abondances.

Nous avons étudié des étoiles Am de différentes températures et luminosités et quelques étoiles Am occupant une position particulière dans des diagrammes photométriques.

Nous avons trouvé pour certaines étoiles un désaccord entre les indices dits d'abondance et les abondances déterminées à grande dispersion.

Il s'agit souvent de géantes, ou de binaires spectroscopiques ou d'étoiles ayant une rotation observable. Cependant, il n'y a pas de relations entre ces différents phénomènes et les désaccords constatés. Nous donnerons quelques exemples d'étoiles que nous avons étudiées.

Les indices d'abondance sont-ils réellement dans tous les cas ce qu'ils sont supposés être? Dans quels cas ne le sont-ils pas et pourquoi? Ces questions restées sans réponse jusqu'à présent devraient être discutées.

Abstract. Our programme involves the study of Am stars of differing temperatures and luminosities as well as of the Am stars deviating from the positions of the classical ones in the photometric diagrams. We have found for certain stars a significant disagreement between the so-called 'abundance indices' and the abundances determined at high dispersion. Often giants or spectroscopic binaries, or stars with a detectable rotation, are involved. However, there are no consistant relationships between these different peculiarities and the observed disagreements. We shall give some examples of stars studied by us. Are these 'abundance indices' really what they are supposed to be in all cases? In which cases are they not and why?

\section{Effets de la binarité sur les indices d'abondances}

Nous avons observé une étoile à fort indice $\Delta m_{1}$, classée Am par Bidelman. Nous avons obtenu, à $12 \AA \mathrm{mm}^{-1}$, un très beau spectre à doubles raies. Dans l'analyse détaillée à grande dispersion, par la méthode des courbes de croissances différentielles, avec les modèles d'atmosphères 'blanketés' de Carbon-Gingerich, l'étoile se révèle être normale. Nous pensons que la binarité est la raison principale du désaccord entre la classification photométrique et l'analyse détaillée d'abondances. En rassemblant les quelques étoiles Am (SB1, SB2) dont les abondances ont été déterminées, on s'aperçoit que la relation $\Delta m_{1} /$ anomalies d'abondances présente de fortes contradictions. 
D'autre part, une estimation rapide de l'influence de la binarité sur les indices de Strömgren $m_{1}$ et $c_{1}$ a permis de mettre en évidence des effets non négligeables sur ces 2 indices. Les photométristes de l'Observatoire de Genève ont traité ce problème pour les indices du système de Genève. En première approximation nous pouvons dire que la binarité spectroscopique peut produire de faux effets d'abondance et de luminosité. Et en conséquence, on ne peut affirmer qu'une étoile est Am parce que $\Delta\left[m_{1}\right] \leqslant-0.025$.

Les photométries comme les classifications à faible dispersion ne sont pas toujours suffisantes pour conclure des anomalies d'abondances d'une étoile. Le problème est d'autant plus important pour les étoiles Am que, statistiquement, elles sont toutes binaires spectroscopiques.

\section{Détermination de l'abondance en Ca des étoiles Am}

Les figures $\Delta\left[m_{1}\right] / \Delta k$ de R. C. Henry (1969) et de R. C. Henry et J. E. Hesser (1971) montrent que le critère classique d'un type spectral $\mathrm{K}$ faible (comparé au type hydrogène) n'est pas capable de mettre en évidence le caractère métallique d'un certain nombre d'étoiles.

Il est intéressant de voir si les analyses spectrophotométriques fines trouvent que le calcium est normal pour ces étoiles Am. Les résultats pour 22 Boo (étoile toujours classée à raies métalliques, ayant un $\Delta\left[m_{1}\right]=-0.05$ comparable à celui de 63 Tau) sont: 22 Boo présente les anomalies d'abondance caractéristiques des étoiles Am; la déficience en Ca est faible mais réelle $\left([\mathrm{Ca} / \mathrm{H}]_{\odot}^{*}=-0.45\right.$ si on utilise les raies de $\mathrm{Ca} \mathrm{I}$ ou les ailes observées de la raie $\mathrm{K}$ comparées aux profils théoriques calculés par Henry). Mais dans le diagramme $\Delta\left[m_{1}\right] / \Delta k$ de Henry, la raie $\mathrm{K}$ serait au contraire normale.

De même pour les autres étoiles étudiées dans le groupe Van 't Veer, nous avons des désaccords qui nous semblent significatifs. Mais plus les etoiles sont froides plus il y a de raies parasites mesurées dans les 2 filtres photométriques de Henry. Ce dernier en tient compte, mais suppose que les raies ionisées parasites se comportent comme la raie $\mathrm{K}$ de Ca II. Aussi, pour les étoiles Am qui ont plus de raies parasites qu'une étoile normale de même type $\mathrm{K}$ (ou $\mathrm{H}$ ), (et même d'autant plus que l'étoile est plus 'métallique'), l'écart à la normale mesuré $\Delta k$ ne traduit pas seulement une raie $\mathrm{K}$ faible (et donc une abondance faible en $\mathrm{Ca}$ ), mais aussi l'exaltation des raies métalliques pour une température et une luminosité données.

Il sera important d'assurer ces résultats pour savoir:

- si les photométries $u b v y, \beta$ et $k$ trient toutes les étoiles Am et elles seulement,

- si il existe ou non des étoiles Am à raie $\mathrm{K}$ normale (la détection des étoiles Am à faible dispersion est-elle donc possible?),

- si Ca peut être normal, ou même un peu surabondant, dans les étoiles Am, i.e. si l'on recherche la cause des étoiles $\mathrm{Am}$, la sous-abondance du $\mathrm{Ca}$ est-elle un problème particulier dans les anomalies d'abondance des étoiles Am et dépend-elle de paramètres et de processus physiques propres au calcium? 


\section{BIBLIOGR APHIE}

Henry, R. C.: 1969, Ap. J. Suppl. 18. 47.

Henry, R. C. and Hesser, J. E.: 1971, Astrophys. J. Suppl. 23, 421.

\section{DISCUSSION}

Rudkjфbing: Greenstein found many years ago that the abundance differences between one metallicline star and some $F$ stars were a function of the ionization potentials, when determined by a curve-ofgrowth method (with the same curve). This, to me, clearly indicates that the differences between a metallic-line star and an F star is just that of the atmospheric structure.

The occurrence of metallic-line stars in the Hyades also seems to rule out an abundance-anomaly explanation.

(Reference may be made to a tentative explanation of the phenomenon put forward about 25 years ago in Annales d'Astrophysique).

Van ' $t$ Veer: We are perfectly conscious of the complexity of the interpretation problems concerming Am stars. But over the last 25 years it has been largely proven by many authors that Am phenomena may be explained by surface abundance anomalies. Reference may be made to the works of these last 15 years: C. van 't Veer, P. S. Conti, F. Praderie, M. Smith. In the last two you can find the new tentative explanation by diffusion processes (cf. also the current work of A. Baglin, S. and G. Vauclair, in Astron. Astrophys.). 